\title{
Diabetic and non-diabetic lumbosacral radiculoplexus neuropathy
}

\author{
Minal J. Bhanushali, Suraj Ashok Muley \\ Departments of Neurology, Emory University, Atlanta, Georgia and University of Minnesota, Minneapolis, Minnesota
}

\begin{abstract}
Background: Lumbosacral radiculoplexus neuropathy (LRPN) originally described in diabetic patients is a distinct clinical condition characterized by debilitating pain, weakness and atrophy most commonly affecting the proximal thigh muscles asymmetrically. The syndrome is usually monophasic and preceded by significant weight loss (at least more than $10 \mathrm{lbs}$ ). Though a self-limited condition, recovery is gradual with some residual weakness. Recent advances and research has provided new insights in the pathogenesis and thereby management of this syndrome. In this paper, we review the clinical and diagnostic features as well as discuss recent insights and treatment strategies along with our experience in the management of patients with diabetic and non-diabetic LRPN. Materials and Methods: Literature in English published between 1953 and 2008 was searched in the MEDLINE and Pubmed database, maintained by the US National library of medicine and National institutes of health, using key words of diabetic amyotrophy, lumbosacral radiculoplexus neuropathy, diabetic proximal neuropathy, diabetic radiculopathy and diabetic lumbosacral plexopathy. In addition, literature reported in various textbooks on peripheral neuropathy was reviewed as well. Observation: The diagnosis relies mostly on clinical suspicion and characteristic electromyographic findings. The exact pathogenesis of the illness remains unknown, but there seems to be a component of immune-mediated inflammatory microvasculitis which causes secondary ischemia of the lumbosacral plexus. This has prompted a trial of immunosuppressive agents (like steroids) with an attempt to alter the course of the illness. A few reports have noted that immunosuppression when instituted early in the course of the illness (within three months of symptom onset) may hasten the recovery and improve symptoms. Conclusion: Though the exact mechanism of LRPN in diabetic and non-diabetic patients remains unknown, new evidence alludes to an underlying inflammatory vasculitic process. Early treatment with immunosuppressants may be beneficial in some cases, although the data available at this time is limited to a small cohort of patients. The decision is individualized weighing
\end{abstract}

the risks and benefits in a given patient. Future research in this direction with double-blinded case-controlled studies is required to investigate this further.

Key words: Diabetic amyotrophy, diagnosis and treatment, lumbosacral plexopathy, lumbosacral radiculoplexus neuropathy, non-diabetic amyotrophy.

\section{Introduction}

Lumbosacral radiculoplexus neuropathy (LRPN) is an uncommon but distinct condition characterized by asymmetrical lower extremity pain, weakness and muscle atrophy affecting commonly the thigh muscles; mild sensory symptoms are seen. Though originally described in patients with diabetes, more recently it has also been recognized in non-diabetic patients. ${ }^{[1]}$ The pathogenesis is thought to be a result of microvasculitis and resultant ischemic injury to the lumbosacral roots, plexus and / or peripheral nerves. ${ }^{[2]}$ A similar syndrome has also been described in the thoracic nerve roots (i.e. thoracic radiculoneuropathy) and the brachial plexus (i.e. cervical radiculoplexus neuropathy).$^{[3]}$ In spite of its often monophasic and subacute course, it can be associated with significant morbidity due to debilitating pain and residual weakness. ${ }^{[4-6]}$

Burns originally described this condition in diabetic patients in $1890 .{ }^{[7]}$ Subsequently, Garland re-described it and coined the terms " diabetic myelopathy" (1953) ${ }^{[8]}$ and "diabetic amyotrophy" (1955). ${ }^{[9,10]}$ Since then, it has been known by various names including the BurnsGarland syndrome, ${ }^{[4]}$ diabetic polyradiculopathy, ${ }^{[5]}$ femoral and femoral-sciatic neuropathy of diabetes, ${ }^{[11,12]}$ proximal diabetic neuropathy, ${ }^{[13]}$ diabetic lumbosacral plexopathy ${ }^{[14]}$ etc. Currently, the term 'diabetic lumbosacral radiculoplexus neuropathy' (DLRPN) is preferred by many authors as it broadly encompasses the various theories regarding anatomical localization and 
pathophysiology. ${ }^{[2]}$ In contrast to DLRPN, LRPN affecting the non-diabetic patients is a recently recognized condition with clinical features, pathogenesis and prognosis similar to its diabetic counterpart. ${ }^{[1,15]}$

\section{Materials and Methods}

Literature in English published between 1953 and 2008 was searched in MEDLINE and Pubmed database maintained by the US National library of medicine and National institutes of health, using key words diabetic amyotrophy, lumbosacral radiculoplexus neuropathy, diabetic proximal neuropathy, diabetic radiculopathy and diabetic lumbosacral plexopathy. In addition, the literature reported in various textbooks for peripheral neuropathy and data presented at the Academy of Neurology annual meeting was reviewed.

\section{Clinical presentation}

The clinical features of DLRPN and LRPN are essentially indistinguishable except for the presence of diabetes mellitus. ${ }^{[15-17]}$

Patients are commonly middle-aged but can be older. Patients with DLRPN reported by Coppack et al., ranged between 46 to 71 years of age at the time of onset of symptoms with mean age being 62 years $^{[18]}$, whereas those reported by Subromony and Wilbourn ranged from 35 to 84 years. ${ }^{[19]}$ Dyck et al., reported 33 cases of DLRPN with onset between 35.8 and 75.9 years with a median age of 65.4 years. ${ }^{[2]}$ In their series of 57 patients with non-diabetic LRPN, median age was about 65 years with the age of onset ranging between 27.8 to 86.5 years. ${ }^{[2]}$ The illness is commonly preceded by unintentional weight loss of more than 10 lbs. ${ }^{[17]}$

In cases of DLRPN, patients usually have Type II diabetes, although in some cases, diabetes comes to light only after the initial presentation of neuropathy. In comparison to patients with diabetic polyneuropathy patients with DLRPN tend to have diabetes that is better controlled and of shorter duration, have lower body mass index, are less likely to be on insulin and have fewer systemic complications like nephropathy or retinopathy. ${ }^{[2]}$ This is consistent with the suggestion that the pathogenesis of DLRPN is independent of the severity and duration of hyperglycemia and the resultant metabolic derangements.

The characteristic presentation is of acute or subacute onset of severe, asymmetrical lower extremity pain and paresthesias involving the anterolateral thigh region. ${ }^{[9,10,18,19]}$ Some patients may report concomitant lower back pain..$^{[2]}$ Pain is described as deep aching, lancinating or burning and tends to be worse at night or on contact with clothes or bed sheets (contact allodynia). ${ }^{[2]}$ Sensory examination reveals hypoesthesia on the anterolateral aspect of the thigh and inner aspect of the leg. ${ }^{[2]}$ Approximately 50\% of patients have concomitant length-dependent sensorimotor polyneuropathy with distal sensory loss. ${ }^{[5,9,10]}$

Pain is followed by weakness that evolves over weeks to months, commonly affecting the proximal thigh (quadriceps and iliopsoas) muscles. Weakness in sciatic distribution is reported ${ }^{[1]}$ but is less common early in the disease compared to the proximal weakness. ${ }^{[13,19]}$ Patients commonly complain of difficulty climbing stairs, rising from low chairs and buckling of the knee with weightbearing. Later in the course of the illness, pain and weakness may spread to distal leg segments (foot drop) and to the contralateral extremity. ${ }^{[8,19,20,21]}$ Early wasting of the quadriceps muscle and absence or reduction of the patellar reflex are common features. ${ }^{[9,10]}$ In addition, during the course of the illness, patients may develop autonomic impairment manifested as orthostatic intolerance and changes in sphincter control. ${ }^{[2]}$ In summary, all classes of nerve fibers including motor, sensory and autonomic tend to be involved.

\section{Diagnostic tests}

A high index of suspicion is important in making a clinical diagnosis. These patients are often misdiagnosed as having compressive lumbosacral polyradiculopathy that is often supported by the "incidental" finding of spondylotic changes in the lumbosacral spine. Acute onset of pain in the area of the thigh with weakness in the femoral distribution and absence of the knee reflex in a diabetic patient can rarely be attributed to compressive radiculopathy. Electrodiagnostic testing may be useful to confirm the diagnosis but if done early in the disease process may be non-diagnostic. The nerve conduction studies in affected nerves show reduced compound muscle action potential (CMAP) amplitudes and absent or reduced sensory responses with proportionate slowing of the conduction velocities indicative of an axonal process. ${ }^{[2,15]}$ The femoral nerve is commonly affected resulting in reduced femoral CMAP amplitude. ${ }^{[19]}$ Approximately 50\% of patients with DLRPN may have a concomitant generalized polyneuropathy and more diffuse electrophysiological abnormalities compared to nondiabetic patients. ${ }^{[5,6,15,19]}$

Electromyography (EMG) of affected muscles shows changes of acute denervation in the form of positive sharp waves and fibrillation potentials. Assessment of the voluntary motor unit action potentials (MUAPs) in the acute phase may reveal reduced recruitment, whereas in chronic cases reinnervation changes consisting of high amplitude, long duration, and polyphasic MUAPs with reduced recruitment are commonly seen. ${ }^{[5,19]}$ The disease process tends to be multifocal and asymmetric. ${ }^{[5]}$ Most severe changes are seen in the lumbar myotomes affecting the quadriceps, iliopsoas and thigh adductors. ${ }^{[19]}$ Lumbar and thoracic paraspinal muscles ${ }^{[15,19]}$ may also show fibrillation potentials. ${ }^{[2,5]} \mathrm{In}$ 
addition, electrophysiological abnormalities may also be seen in segments that are not clinically involved including cervical or lumbosacral segment. ${ }^{[15,19]}$

Abnormal quantitative sensory and autonomic testing in the affected lower extremity can be seen in both DLRPN and LRPN but is rarely useful in making a diagnosis. ${ }^{[2]}$

Similarly, laboratory testing shows changes consistent with diabetes but no other specific diagnostic clues. Patients with DLRPN have increased levels of random blood glucose and glycosylated hemoglobin levels. Also, a significant number of patients with LRPN and normal fasting blood glucose levels may have impaired glucose tolerance either at the time of presentation or a few months after diagnosis. ${ }^{[22]}$ In addition, erythrocyte sedimentation rates (ESR) may be elevated in both forms of LRPN. ${ }^{[14,15]}$ Occasionally, patients may have reactive rheumatoid factor and antinuclear antibodies which are of uncertain relationship to the disease. ${ }^{[15]}$

Cerebrospinal fluid (CSF) protein may be elevated, especially if there is involvement of the nerve roots. A median value of $89.5 \mathrm{mg} / \mathrm{dl}$ (range, 44-214 mg/dl) in the DLRPN cohort and $66.5 \mathrm{mg} / \mathrm{dl}$ (range, $18-283 \mathrm{mg} / \mathrm{dl}$ ) in the non-diabetic LRPN patients has been reported. ${ }^{[2]}$

Radiological investigations such as magnetic resonance imaging (MRI) of the lumbosacral spine and plexus are useful in ruling out other potential etiologies such as compressive polyradiculopathy, infiltrative plexopathy or compression of the plexus by a hematoma, tumor mass etc. Nevertheless, enhancement of lumbosacral nerve roots and plexus ${ }^{[23]}$ may also be seen in both DLRPN and LRPN.

\section{Pathogenesis}

The understanding of the pathogenetic mechanisms of DLRPN has evolved over time. Earlier authors believed that the metabolic derangement and vasculopathy that occurs in diabetes played a major role in pathogenesis. ${ }^{[13,24,25]}$ Recent studies indicate that the pathogenesis of DLRPN is independent of these metabolic factors and that it is primarily an immune-mediated process. However, the question whether metabolic derangement in diabetes alters the blood nerve barrier, thereby making it more susceptible to immune-mediated injury remains unresolved. ${ }^{[26,27]}$

Pathological findings in distal cutaneous sensory nerves (sural, superficial peroneal and saphenous) ${ }^{[14,16,17,28]}$ and proximal cutaneous nerves (intermediate cutaneous nerve of the thigh ${ }^{[29,30]}$ reported in various studies primarily show multifocal areas of patchy inflammation or vasculitis affecting small caliber neural blood vessels, secondary nerve ischemia with axonal degeneration and focal demyelination in the affected area. Given the chronic and progressive pathogenesis, changes in the nerve consist of areas of active axonal degeneration, empty nerve strands, perineural scarring as well as regeneration changes in the form of injury neuroma and epineurial neovascularization. ${ }^{[17]}$ The detailed findings are summarized and listed in Table 1.

In addition to this, the role of inflammatory pathogenesis has been further studied by Kelkar et al., ${ }^{[29]}$ who reported that immune-complex and complement deposits are seen at the site of inflammation, and by Kawamura et al. ${ }^{[31]}$ who investigated inflammatory mediators like intercellular adhesion molecule-1 (ICAM-1), tumor necrosis factor $\alpha$ (TNF- $\alpha$ ), interleukin 6 (IL-6) and neural factor $\kappa \mathrm{B}(\mathrm{NF}-\kappa \mathrm{B})$ in the sural nerve biopsies of 19 patients with DLRPN and 13 patients with non-diabetic LRPN as compared to 20 control subjects. They found upregulation of inflammatory markers ICAM-1, TNF- $\alpha$, and NK- $\mathrm{BB}$ in patients with LRPN, with expression pattern similar to, but less florid than neuropathy associated with systemic vasculitis. Another interesting feature noted was that three patients with higher TNF- $\alpha$ expression clinically had severe pain and acute presentation, which responded well to methylprednisolone. ${ }^{[31]}$ The pathogenesis and pathological changes reported in LRPN are similar to those seen in DLRPN. ${ }^{[16,31]}$

\section{Management}

Traditionally, the approach towards the management of DLRPN consisted of symptomatic treatment of pain and tight glycemic control. ${ }^{[18,32]}$ Part of this was due to reports of eventual recovery of weakness over time and partly due to lack of adequate understanding of the pathophysiology. However, there has been greater recognition recently that the disease can evolve over months with disabling pain and residual weakness. Also, presently the pathogenesis is better understood and appears to be patchy microvasculitis and secondary nerve ischemia, in contrast to the traditionally held belief of it being a metabolic dysfunction of the peripheral nervous system. This has led to the investigation of various immunosuppressive treatment strategies to alter the course of DLRPN.

\section{Immunosuppressants and DLRPN}

Various immunosuppressive treatments in the form of prednisone, prednisone in combination with other immunosuppresants (like azathioprine or cyclophosphamide), and intravenous immunoglobulin (IVIG) have been reported with variable outcomes. Preliminary studies were done by Bradley et al., ${ }^{[14]}$ assessing the response to prednisone in three patients and prednisone with cyclophosphamide in two patients with DLRPN. They reported improvement of pain in four and improvement of weakness in two patients.

Similarly, Krendel et al., ${ }^{[33]}$ treated 13 patients with prednisone or IVIG either alone or in combination with azathioprine or cyclophosphamide. All symptoms stopped worsening and there was improvement in 


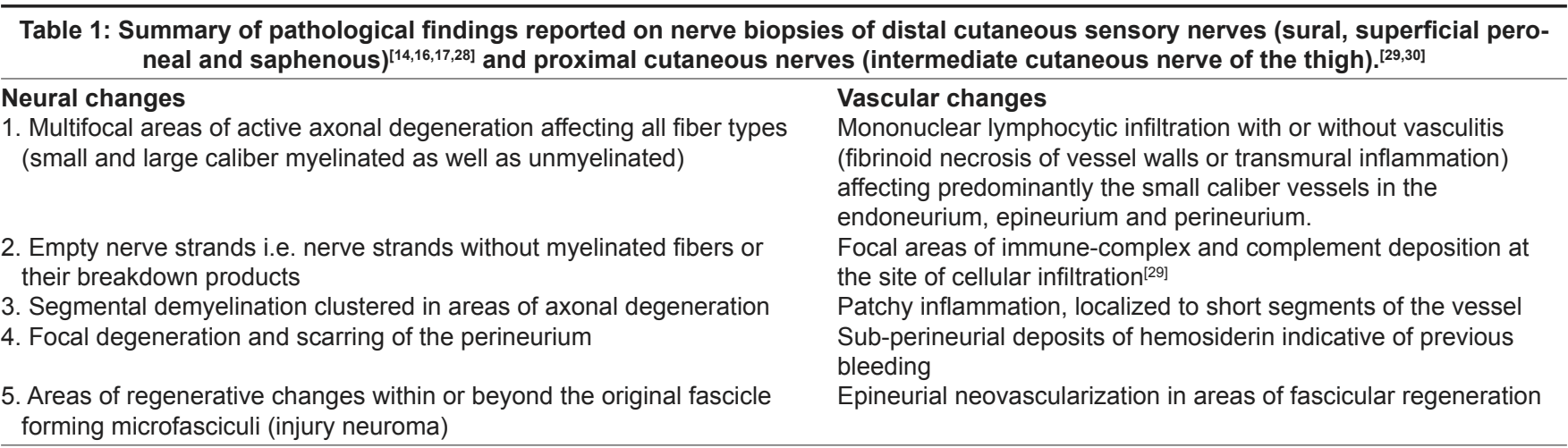

strength and activities of daily living in the majority of patients. The interval between the onset of the disease and the institution of the treatment was not mentioned. A retrospective study ${ }^{[16,34]}$ assessing the use of oral or intravenous pulsed methylprednisolone $500 \mathrm{mg}$ for two days every two weeks for up to three months within one week to five months of onset in nine patients reported dramatic improvement of pain and weakness in seven patients treated within three months of symptom onset. In patients treated at four and five months, improvement was delayed and efficacy was uncertain.

These preliminary efforts prompted institution of a randomized double-blinded multicenter trial, investigating the role of intravenous methylprednisolone in the treatment of DLRPN. ${ }^{[35]}$ A significant difference in the primary outcome measure (neuropathy impairment score of the lower limb) was not found, suggesting a lack of efficacy. The lack of efficacy may have been from delay in institution of the treatment in this multicenter study. Even so, there was improvement in pain and positive neuropathic symptoms in the treatment group. A study by Kilfoyle et al., ${ }^{[34]}$ suggests that treatment with pulsed methylprednisolone instituted within three months of symptom onset leads to significant improvement in pain and weakness in the majority of patients. Response to pain occurred within days to weeks and weakness stabilized and improved faster than the natural history of the disease. Treatment after three months was of uncertain benefit but should be tried in patients with progressive weakness or intractable pain.

Regarding the immunosuppressive strategies for nondiabetic LRPN, the data is limited to a few case reports or small patient cohorts. Awerbuch et al., reported no response to prednisone in one patient with idiopathic relapsing LRPN, ${ }^{[36]}$ whereas Verma and Bradley ${ }^{[37]}$ reported improvement with high-dose IVIG $(0.8 \mathrm{~g} / \mathrm{kg} /$ day for five days) in a patient with progressive lumbosacral plexopathy who did not respond to prednisone, regular dose of IVIG or plasmapheresis. Triggs et al. ${ }^{[38]}$ reported improvement of pain and weakness in four patients with idiopathic LRPN with regular dose IVIG ( $0.4 \mathrm{~g} / \mathrm{kg} / \mathrm{day}$ for five days) and in one patient with high-dose IVIG
$(0.8 \mathrm{~g} / \mathrm{kg} /$ day for three days) at a mean of 3.8 months from onset. Lastly, Dyck et al., ${ }^{[39]}$ reported 10 patients treated with intravenous methylprednisolone and one patient treated with prednisone at a comparable dose with improvement in pain, weakness and ability to ambulate suggesting efficacy. Treatment was instituted at a median of five months from onset (range 1-48 months).

\section{Role of metabolic factors and treatment}

Originally, DLRPN was thought to be related to metabolic derangement and treatment strategies emphasized aggressive glycemic control. However, Bradley et al., ${ }^{[14]}$ reported continued worsening of DLRPN in three patients despite adequate control of diabetes. Other authors as well, reported variable responses to aggressive diabetic control, ranging from marked improvement to significant residual disability. ${ }^{[24,32]}$

\section{Symptomatic management}

Given the disabling pain, patients often require narcotic analgesics in the acute phase with or without other agents used in chronic neuropathic pain like anti-epileptics and tricyclic antidepressants. Physical therapy may be beneficial after the progressive phase of the illness. ${ }^{[26]}$

\section{Natural course of IIIness and prognosis}

Coppack et al., ${ }^{[18]}$ studied the natural course of DLRPN in 27 patients and reported spontaneous recovery in all patients. Mean time to recovery was about three months (range 1-12 months) and recovery was generally complete by 18 months. Pain was the first symptom to remit, usually within a few weeks or months. This was followed by a gradual improvement in weakness that was at times incomplete with residual thigh weakness. Despite residual weakness, disability was minor in the majority of patients. Pascoe et al., ${ }^{[6]}$ studied 21 patients for about three years from the onset of symptoms and found 12 had resumed normal walking, seven ambulated with an aid and two were wheelchair-bound. Recurrence or relapse of the illness 
can occur but is uncommon. ${ }^{[5,18]}$ The natural course and prognosis of non-diabetic LRPN is identical to that of DLRPN. ${ }^{[2]}$

\section{Future research}

Given the paucity of data for treatment of LRPN, a key issue that remains unanswered is the impact of immunosuppression in the acute phase of illness on modifying the disease course, alleviating pain and minimizing residual weakness and disability. This also raises the question of possible ways to better stratify patients who will benefit the most (with minimal adverse effects) as well as optimal intensity and strategies (prednisone vs. IVIG vs. other agents) for immunosuppression. Further randomized controlled clinical trials aimed to answer these questions are warranted.

\section{Conclusion}

DLRPN and non-diabetic LRPN is a self-limiting condition. Newer insights into the pathogenesis allude to an underlying immune-mediated process. Early institution of immunosuppressive treatment may have a potential role in the management of DLRPN and LRPN. However, the data is limited to small patient cohort studies and case reports. There have been no randomized controlled clinical trials or large cohort studies to demonstrate its efficacy adequately. Hence, the decision to initiate immunosuppression should be individualized considering the clinical presentation, relative acuity and time between symptom onset (less than three months), extent of disability and severity of symptoms, as well as the risks and benefits in a given patient. Further controlled studies analyzing the role of early treatment with immunosuppressant medications are needed to establish its role with certainty.

\section{References}

1. Evans BA, Stevens JC, Dyck PJ. Lumbosacral plexus neuropathy. Neurology 1981;1:1327-30.

2. Dyck PJ, Windebank AJ. Diabetic and nondiabetic lumbosacral radiculoplexus neuropathies: new insights into pathophysiology and treatment. Muscle Nerve 2002;25:477-91.

3. Dyck P. Radiculoplexus neuropathies: diabetic and nondiabetic varieties. 4th ed. Philadelphia: Elvsevier; 2005.

4. Barohn RJ, Sahenk Z, Warmolts JR, Mendell JR. The Bruns-Garland syndrome (diabetic amyotrophy). Revisited 100 years later. Arch Neurol 1991;48:1130-5.

5. Bastron JA, Thomas JE. Diabetic polyradiculopathy: clinical and electromyographic findings in 105 patients. Mayo Clin Proc $1981 ; 56: 725-32$.

6. Pascoe MK, Low PA, Windebank AJ, Litchy WJ. Subacute diabetic proximal neuropathy. Mayo Clin Proc 1997;72:1123-32.

7. L B. Ueber neuritische beim diabetes mellitus. Berl Klin Wochenscher $1890 ; 509-15$.
8. Garland H, Taverner D. Diabetic myelopathy. Br Med J 1953:1405-8.

9. Garland H. Diabetic amyotrophy. Br Med J 1955;2:1287-90.

10. Garland H. Diabetic amyotrophy. Br J Clin Pract 1961;15:9-13.

11. Calverley JR, Mulder DW. Femoral neuropathy. Neurology 1960;10:963-7.

12. Gydell K, Skanse B. A rare type of femoral-sciatic neuropathy in diabetes mellitus. Acta Med Scand 1956;155:463-8.

13. Asbury AK. Proximal diabetic neuropathy. Ann Neurol 1977;2:179-80.

14. Bradley WG, Chad D, Verghese JP, Liu HC, Good P, Gabbai AA, et al. Painful lumbosacral plexopathy with elevated erythrocyte sedimentation rate: a treatable inflammatory syndrome. Ann Neurol 1984;15:457-64.

15. Dyck PJ, Norell JE, Dyck PJ. Non-diabetic lumbosacral radiculoplexus neuropathy: natural history, outcome and comparison with the diabetic variety. Brain 2001;124:1197-207.

16. Dyck PJ, Engelstad J, Norell J, Dyck PJ. Microvasculitis in nondiabetic lumbosacral radiculoplexus neuropathy (LSRPN): similarity to the diabetic variety (DLSRPN). J Neuropathol Exp Neurol 2000;59:525-38.

17. Dyck PJ, Norell JE, Dyck PJ. Microvasculitis and ischemia in diabetic lumbosacral radiculoplexus neuropathy. Neurology 1999;53:2113-21.

18. Coppack SW, Watkins PJ. The natural history of diabetic femoral neuropathy. Q J Med 1991;79:307-13.

19. Subramony SH, Wilbourn AJ. Diabetic proximal neuropathy. Clinical and electromyographic studies. J Neurol Sci 1982;53:293-304.

20. Locke S, Lawrence DG, Legg MA. Diabetic amyotrophy. Am J Med $1963 ; 34: 775-85$

21. Goodman JI. Femoral neuropathy in relation to diabetes mellitus: report of 17 cases. Diabetes 1954;3:266-73.

22. Kelkar P, Hammer-White S. Impaired glucose tolerance in nondiabetic lumbosacral radiculoplexus neuropathy. Muscle Nerve 2005;31:273-4.

23. Ishii K, Tamaoka A, Shoji S. MRI of idiopathic lumbosacral plexopathy. Neurology 2004; E6.

24. Chokroverty S, Sander HW. AAEM case report \#13: diabetic amyotrophy. Muscle \& nerve 1996;939-45.

25. Gregersen G. Diabetic amyotrophy--a well-defined syndrome? Acta Med Scand 1969;185:303-10.

26. Bashar katirji HK, David Preston, Robert Ruff, Barbara Shapiro, Neuromuscular disorders in clinical practice: Butterworth Heinemann; 2002.

27. Spencer PS, Weinberg HJ, Raine CS, Prineas JW. The perineurial window--a new model of focal demyelination and remyelination. Brain Res 1975;96:323-9.

28. Llewelyn JG, Thomas PK, King RH. Epineurial microvasculitis in proximal diabetic neuropathy. J Neurol 1998;245:159-65.

29. Kelkar P, Masood M, Parry GJ. Distinctive pathologic findings in proximal diabetic neuropathy (diabetic amyotrophy). Neurology 2000;55:83-8.

30. Said G, Goulon-Goeau C, Lacroix C, Moulonguet A. Nerve biopsy findings in different patterns of proximal diabetic neuropathy. Anna Neurol 1994;35:559-69.

31. Kawamura N, Dyck PJ, Schmeichel AM, Engelstad JK, Low PA, Dyck PJ. Inflammatory mediators in diabetic and non-diabetic lumbosacral radiculoplexus neuropathy. Acta Neuropathol 2008;115:231-9.

32. Casey EB, Harrison MJ. Diabetic amyotrophy: a follow-up study. Br Med J 1972;1:656-9.

33. Krendel DA, Costigan DA, Hopkins LC. Successful treatment of neuropathies in patients with diabetes mellitus. Arch Neurol 1995;1053-61.

34. Kilfoyle DM, ChB ; Kelkar, P. MD; Parry, G. J. MB, ChB, FRACP Pulsed Methylprednisolone Is a Safe and Effective Treatment for Diabetic Amyotrophy. Journal of Clinical Neuromuscular Disease 2003;168-70.

35. Dyck PJ ObP, Bosch PE, Grant I, Burns T, Windebank A, Klein C, Hausbenschild J, Peterson D, Norell J, Capelle S, Lodermeier K, Dyck P S27.005. platform presentation in American academy of Neurology annual meeting, 2006.

36. Awerbuch GI, Nigro MA, Sandyk R, Levin JR. Relapsing lumbosacral plexus neuropathy. Report of two cases. Eur Neurol 1991;31:348-51. 
37. Verma A, Bradley WG. High-dose intravenous immunoglobulin therapy in chronic progressive lumbosacral plexopathy. Neurology 1994;44:248-50.

38. Triggs WJ, Young MS, Eskin T, Valenstein E. Treatment of idiopathic lumbosacral plexopathy with intravenous immunoglobulin. Muscle \& nerve 1997:244-6.

39. Dyck PJ, Norell JE, Dyck PJ. Methylprednisolone may improve lumbosacral radiculoplexus neuropathy. Can J Neurol Sci 2001;28:224-7

Accepted on 10-06-2008

Source of Support: Nil, Conflict of Interest: None declared.

\section{Author Help: Online Submission of the Manuscripts}

Articles can be submitted online from http://www.journalonweb.com. For online submission articles should be prepared in two files (first page file and article file). Images should be submitted separately.

1) First Page File:

Prepare the title page, covering letter, acknowledgement, etc., using a word processor program. All information which can reveal your identity should be here. Use text/rtt/doc/pdf files. Do not zip the files.

2) Article file:

The main text of the article, beginning from Abstract till References (including tables) should be in this file. Do not include any information (such as acknowledgement, your names in page headers, etc.) in this file. Use text/rtt/doc/pdf files. Do not zip the files. Limit the file size to $400 \mathrm{~kb}$. Do not incorporate images in the file. If file size is large, graphs can be submitted as images separately without incorporating them in the article file to reduce the size of the file.

3) Images:

Submit good quality color images. Each image should be less than $1024 \mathbf{~ k b ~ ( 1 ~ M B ) ~ i n ~ s i z e . ~ S i z e ~ o f ~ t h e ~ i m a g e ~ c a n ~ b e ~ r e d u c e d ~ b y ~ d e c r e a s i n g ~}$ the actual height and width of the images (keep up to about 6 inches and up to about 1200 pixels) or by reducing the quality of image. JPEG is the most suitable file format. The image quality should be good enough to judge the scientific value of the image. Always retain a good quality, high resolution image for print purpose. This high resolution image should be sent to the editorial office at the time of sending a revised article.

4) Legends:

Legends for the figures/images should be included at the end of the article file. 